\title{
Clinical Features and Risk Factors Analysis for Hemorrhage in Adults on ECMO
}

\author{
Wenwen $\mathrm{Hu}^{1}$, Meifang Wang${ }^{2}$, Wei Chen ${ }^{2}$, Lin Chai ${ }^{2}$, Elaine Lai-Han Leung ${ }^{3}$, and Yijun \\ Tang $^{2}$ \\ ${ }^{1}$ Postgraduate Training Basement of Jinzhou Medical University, Taihe Hospital \\ ${ }^{2}$ Taihe Hospital \\ ${ }^{3}$ Macau University of Science and Technology
}

November 24, 2020

\begin{abstract}
Background: The use of extracorporeal membrane oxygenation (ECMO) to support critically ill patients with cardiorespiratory dysfunction is increasing over the last decades. However, hemorrhagic complications remain occurring frequently during ECMO support, which have a significant impact on morbidity and mortality. Methods: A retrospective study was performed on the 60 patients, who were admitted to the Taihe hospital in Shiyan City, Hubei Province from February 2017 to October 2020. All those were rescued with ECMO. Including 18 patients developed hemorrhage complications and 42 patients did not. Demographic, laboratory tests, clinical manifestations prior to ECMO were collected to analysis the clinical features. Univariable and multivariable logistic analysis methods were used in our study to explore the risk factors for hemorrhage in adults on ECMO. Results: There were significant differences between the hemorrhage group and no-hemorrhage group in duration of ECMO support, mode of ECMO, red blood cell count, hemoglobin, platelet count, serum creatinine. Particularly, multivariate logistic analysis showed that the longer duration of ECMO support and the higher activated partial thromboplastin time (APTT) prior to ECMO were independent factors for hemorrhage in adults on ECMO. In addition, we found that the mortality of hemorrhagic patients was higher than no-hemorrhagic patients. Cannula site was the most common bleeding site. Most bleeding events occurred within the first three days of ECMO therapy. Conclusions: Clinicians should evaluate the risk of hemorrhage based on patients' coagulation function, underlying disease as well as the duration of ECMO support. Especially in the first three days during ECMO support. Attempting to wean from ECMO early whenever feasible is also effective to reduce the occurrence of hemorrhage. Special attention should be given on cannula site, mucosal, dermal and digestive tract to alert hemorrhage.
\end{abstract}

\section{Hosted file}

Manuscript.pdf available at https://authorea.com/users/378513/articles/494983-clinicalfeatures-and-risk-factors-analysis-for-hemorrhage-in-adults-on-ecmo 

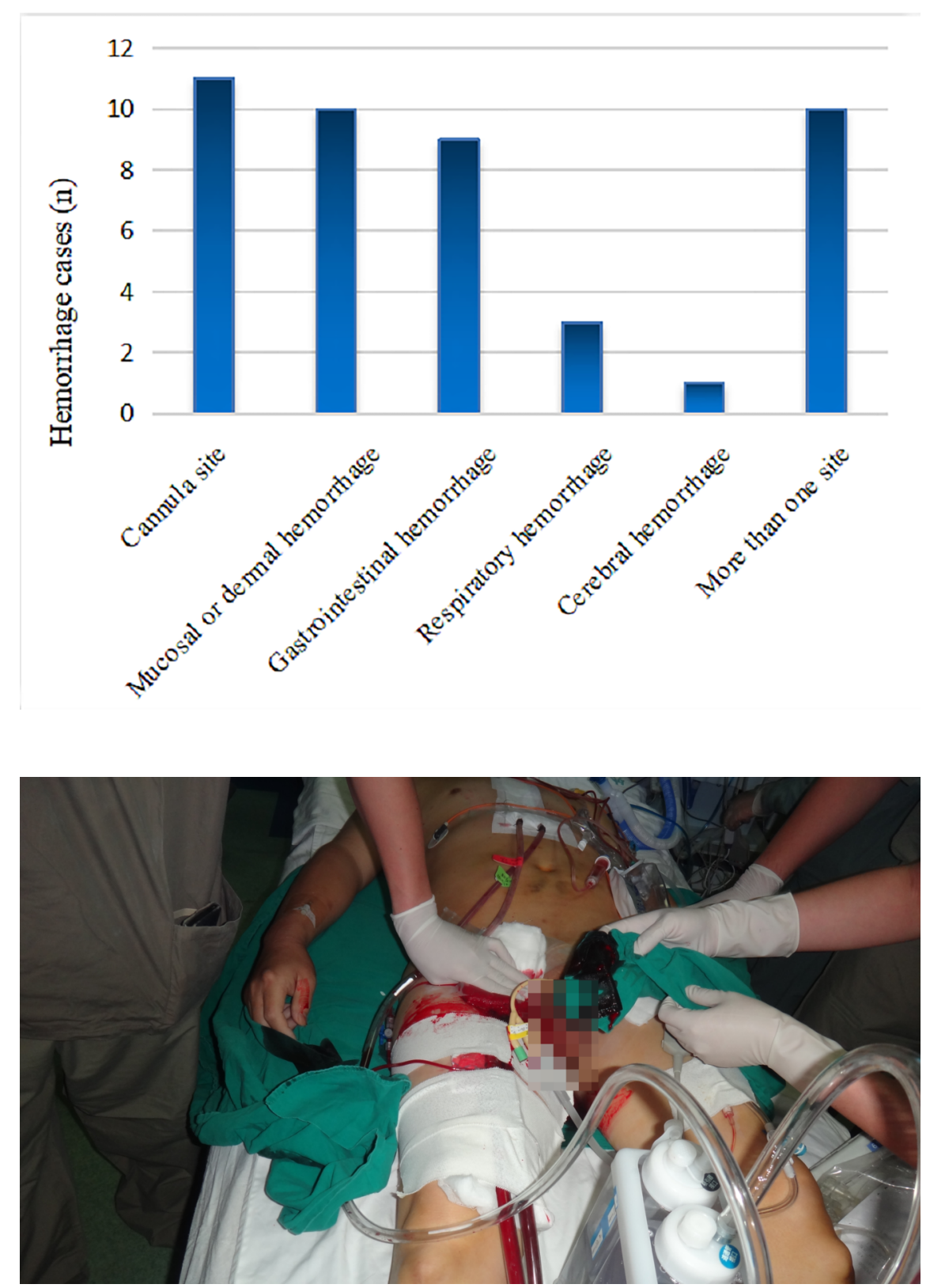

\section{Hosted file}

Tables.pdf available at https://authorea.com/users/378513/articles/494983-clinical-featuresand-risk-factors-analysis-for-hemorrhage-in-adults-on-ecmo 